OPEN ACCESS

Edited by:

Ying Ma,

University of Coimbra, Portugal

Reviewed by:

Munusamy Madhaiyan

Temasek Life Sciences Laboratory,

Singapore

Collin M. Timm

Applied Physics Laboratory, Johns Hopkins University, United States

*Correspondence:

Daria Rybakova

daria.rybakova@tugraz.at

${ }^{\dagger}$ These authors have contributed equally to this work.

Specialty section: This article was submitted to

Plant Microbe Interactions, a section of the journa Frontiers in Plant Science

Received: 04 May 2017 Accepted: 07 July 2017 Published: 27 July 2017

Citation:

Rybakova D, Rack-Wetzlinger U, Cernava T, Schaefer A, Schmuck $M$ and Berg G (2017) Aerial Warfare: A Volatile Dialogue between the Plant Pathogen Verticillium longisporum and Its Antagonist Paenibacillus polymyxa.

Front. Plant Sci. 8:1294. doi: 10.3389/fpls.2017.01294

\section{Aerial Warfare: A Volatile Dialogue between the Plant Pathogen Verticillium longisporum and Its Antagonist Paenibacillus polymyxa}

\author{
Daria Rybakova ${ }^{1 * \dagger}$, Ute Rack-Wetzlinger ${ }^{1 \dagger}$, Tomislav Cernava ${ }^{2}$, Angelika Schaefer ${ }^{1}$, \\ Maria Schmuck ${ }^{1}$ and Gabriele Berg ${ }^{1}$ \\ ${ }^{1}$ Institute of Environmental Biotechnology, Graz University of Technology, Graz, Austria, ${ }^{2}$ Austrian Centre of Industrial \\ Biotechnology - GmbH, Graz, Austria
}

Verticillium wilt caused by Verticillium spp. results in severe yield losses in a broad range of crops. Verticillium outbreaks are challenging to control, and exacerbated by increases in soil temperatures and drought associated with global warming. Employing natural antagonists as biocontrol agents offers a promising approach to addressing this challenge. Paenibacillus polymyxa Sb3-1 was proven to reduce the growth of Verticillium longisporum during in vitro experiments and was shown to promote the growth of oilseed rape seedlings infested with $V$. longisporum. Our novel approach combined in vitro and in planta methods with the study of the mode of interaction between Sb3-1 and V. longisporum EVL43 via their volatile organic compounds (VOCs). Volatile and soluble substances, produced by both microorganisms as a reaction to one another's VOCs, were detected by using both gas and liquid chromatography-mass spectrometry. P. polymyxa Sb3-1 continually produced antimicrobial and plant growth promoting VOCs, such as 2-nonanone and 3-hydroxy-2-butanone. Several other antimicrobial volatile substances, such as isoamyl acetate and durenol, were downregulated. The general metabolic activity of Sb3-1, including protein and DNA biotransformations, was upregulated upon contact with EVL43 VOCs. V. longisporum increased its production of antimicrobial substances, such as 1-butanol, and downregulated its metabolic activities upon exposure to Sb3-1 VOCs. Additionally, several stress response substances such as arabitol and protein breakdown products (e.g., L-Isoleucyl-L-glutamic acid), were increased in the co-incubated samples. The results obtained depict an ongoing dialog between these microorganisms resulting in growth inhibition, the slowing down of metabolism, and the cell death of $V$. longisporum due to contact with the $P$. polymyxa Sb3-1 VOCs. Moreover, the results indicate that VOCs make a substantial contribution to the interaction between pathogens and their natural antagonists and have the potential to control pathogens in a novel, environmentally friendly manner.

Keywords: biocontrol, Verticillium wilt, Verticillium longisporum, Paenibacillus polymyxa, volatile assay, VOCs, plant-microbe interactions, adaptation 


\section{INTRODUCTION}

Verticillium wilt caused by Verticillium spp. is a serious fungal plant disease that affects up to 250 hosts, including many economically important crops such as potato, strawberry, alfalfa, oilseed crops, and a variety of tree species (Hiemstra, 1998; Pegg and Brady, 2002). While V. dahliae Kleb. (1913) and V. alboatrum Reinke and Berthold (1879) attack a high number of host species, V. longisporum (C. Stark) Karapapa et al. (1997) has a more defined host range, primarily infesting cruciferous species (Brassicaceae). V. longisporum is the main contributor to Verticillium wilt in oilseed crops (Zeise and von Tiedemann, 2002; Depotter et al., 2016), and causes up to $50 \%$ of yield losses (Daebeler et al., 1988; Zeise and Steinbach, 2004). Verticillium spp. are the most challenging types of phytopathogens to control due to the extensive viability of their microsclerotia (resting structures), their broad host range, and the genetically heterogeneous and polyphyletic character of Verticillium isolates, as well as the absence of effective fungicide treatments (Fradin et al., 2009; Jiménez-Gasco et al., 2014). Due to the decrease in crop rotation time and global warming (Houghton et al., 2001), the number of disease incidents is expected to increase in the future (Heale and Karapapa, 1999; Siebold and von Tiedemann, 2012). Verticillium has an optimum temperature of $20^{\circ} \mathrm{C}$ and above for most of its life cycle stages (Siebold and von Tiedemann, 2012). In this context, global warming will increase the temperature of the soil's upper layers in which the fungal inoculum is found to be present (Zhang et al., 2005), causing the shifting of soil temperatures toward the biological optimum temperature for the fungus at locations where the disease is currently not a problem. Such temperature-related shifts, or 'fingerprints' in species distribution due to global warming have been observed within a large number of species (Root et al., 2003). In addition, the infection period can be extended at higher temperatures (Dunker et al., 2008). This concurs with recent observations that the wilt disease of the oilseed rape (OSR) has been occurring more frequently in recent, warmer years (Siebold and von Tiedemann, 2012). The Verticillium wilt of strawberry crops is also affected by temperature increases, as was shown by an increase of damage occurring during warm conditions (Schubert et al., 2009). Considering the current trend in plant disease control toward sustainable agriculture and the urgent need for agricultural adaptation to climate change (Howden et al., 2007), environmentally friendly solutions for Verticillium wilt problems, such as biological control, are especially desirable.

Several biocontrol agents (BCAs) such as Serratia plymuthica HRO-C48 (Müller and Berg, 2008), non-pathogenic Verticillium strains (França et al., 2013), as well as several strains of Paenibacillus and Serratia (Rybakova et al., 2016b) were proposed as potentially effective biocontrol agents against $V$. longisporum. Endophytic Paenibacillus strains are known for their plant growth promoting and biocontrol properties (Rybakova et al., 2016a). P. polymyxa Sb3-1 was shown to be particularly active against $V$. longisporum in vitro. This strain demonstrated a plant growth promoting effect on the oilseed rape seedlings in sterile soil, and was deleterious to the seedlings under sterile soil-free conditions (Rybakova et al., 2016b). Several modes of actions are suggested for Paenibacillus spp. as biocontrol agents. They are able to protect plants against pathogenic organisms in a variety of ways including the production of a variety of antimicrobials and insecticides and triggering a plant's defense system known as induced systemic resistance (Grady et al., 2016). Paenibacillus spp. can also produce a biofilm around plant roots that functions as a protective layer preventing pathogens from accessing plant tissue (Timmusk et al., 2005). Recently, the emission of antimicrobial volatile organic substances (VOCs) has been proposed as an important defense mechanism of Paenibacillus (Rybakova et al., 2016a). A multitude of interactions between organisms is based on the emission and perception of volatiles (Wenke et al., 2010). In fact, recent data suggests that the majority of all explored interactions is based on volatile compounds rather than on non-volatile ones (Kanchiswamy et al., 2015). Even plants can sense VOCs produced by neighboring plants (Baldwin et al., 2006; Dicke et al., 2009). Volatiles act as an important medium for interactions between bacteria and fungi below ground (Insam and Seewald, 2010; Effmert et al., 2012). In this context, it was shown that fungi emit a broad spectrum of VOCs with diverse ecological functions affecting both bacteria (Schmidt et al., 2015; Hacquard, 2017) and plants (Ditengou et al., 2015). Bacteria are known to produce highly diverse volatiles (Schulz and Dickschat, 2007). Such microbial VOCs can directly or indirectly mediate increases in the biomass of the host plant. They also increase disease resistance and abiotic stress tolerance, and thereby help plants to combat pathogens (Liu and Zhang, 2015). In terms of microbial interplay it is hypothesized that VOCs can be used either as infochemical molecules affecting the gene expression in the responding microorganism, or as competitive tools, providing an advantage by suppressing or eliminating potential enemies (Kai et al., 2007; Schmidt et al., 2015; Ossowicki et al., 2017). The diversity of soil bacteria and the presence of some less abundant soil bacteria, such as Paenibacillus, play important roles in the production of antifungal volatiles by soil bacterial communities (Hol et al., 2015; Schulz-Bohm et al., 2015). Diazine derivatives produced by several Paenibacillus spp., for example, are able to suppress the growth of bacteria, fungi and yeast (Cernava, 2012). Due to their great potential for application as a pathogen control in industrial environments a patent was filed for commercial utilization of novel bioactive compounds (Aichner et al., 2012). Currently, various tools are available to access bioactivity of microbial volatiles. These tools include pre-screening methods such as split-plate experiments and parallelized VOCs assays (Ryu et al., 2003; Cernava et al., 2015a). Further characterization of the substances involved requires an analytical approach. Highly concentrated volatiles can be captured with an air tight syringe (headspace method) and injected into a GC-MS system. Alternatively, headspace solid-phase microextraction (SPME) can also capture less abundant volatiles, which is beneficial when general VOC emissions of microorganisms are studied (Zhang and Pawliszyn, 1993).

While in recent years the diversity of microbial volatiles has been studied intensively, the ways in which they contribute to the mode of action of biocontrol agents, and their exact role in specific microbe-microbe interactions remain largely 
unknown. In order to address this knowledge gap, we studied the interaction between plant pathogenic Verticillium longisporum EVL43 and the potential biocontol agent Paenibacillus polymyxa Sb3-1 via their volatiles in vitro and in planta. The main objective of the study was to understand the mode of action of the predicted biocontrol agent $P$. polymyxa Sb3-1 against plant pathogen $V$. longisporum via its volatiles. We hypothesized that the exchange of volatiles plays an important role in the interaction between these two microorganisms and is involved in the antagonistic effect of $P$. polymyxa Sb3-1 against Verticillium wilt.

\section{MATERIALS AND METHODS}

\section{Bacterial Strains and Growth Conditions}

The fungal pathogen used was V. longisporum (C. Stark) Karapapa et al. (1997) strains ELV25 and EVL43 from the collection TU Graz, Environmental Biotechnology, described in Messner et al. (1996). V. longisporum ELV25 was grown either on potato dextrose agar (PDA) or in Czapek Dox liquid culture (Sigma-Aldrich). P. polymyxa Sb3-1 (Köberl et al., 2013) as well as its rifampicillin resistant mutant $P$. polymyxa Sb3-1 rif $^{\mathrm{R}}$ (this study) were routinely grown on Standard I nutrient agar (NA, SIFIN, Berlin, Germany) at $30^{\circ} \mathrm{C}$. When required, rifampicin was added at concentrations of $100 \mu \mathrm{g} \mathrm{ml}^{-1}$. For the direct dual culture assays, Reasoner's 2A agar (R2A) (Roth, Karlsruhe, Germany), water yeast agar (WAY), and PDA were used.

\section{Evaluation of the Plant Growth Promotion (PGP) and Biocontrol Effects of $P$. polymyxa Sb3-1 In planta}

Oilseed rape (Brassica napus L. "Traviata H 605886"; KWS Saat Einbeck, Germany) seeds were treated with two concentrations of P. polymyxa Sb3-1 $\left(\log _{10} 7\right.$ and $\left.\log _{10} 5 \mathrm{CFU} \mathrm{ml}{ }^{-1}\right)$ applied to the seeds using the pelleting method according to the protocol described by Müller and Berg (2008). For the PGP studies, the experiment was performed in 5 replicates with 9 seedlings each (3 seeds per pot). The 2-week old seedlings were harvested and their fresh weight was compared to the untreated control. The bacterial abundance on the seeds and on the roots was estimated as described by Rybakova et al. (2016b). For the biocontrol studies, the 1-week old seedlings were inoculated with $V$. longisporum ELV25 using the root dipping method. The experimental setup included 15 pots per treatment with one plant per pot and replicate. The roots of the 1-week old seedlings were artificially injured using a scalpel. The seedlings were inoculated by dipping their roots for $30 \mathrm{~min}$ into $200 \mathrm{ml}$ of Czapek-Dox broth with a 1-week old culture of $V$. longisporum ELV25 adjusted to $10^{6} \mathrm{CFU} \mathrm{ml} \mathrm{ml}^{-1}$. Control treatments were treated the same way as the inoculated seedlings, however they were immersed in sterile water instead of V. longisporum ELV25 blastospore solution. Finally, all plants were transplanted back to the pots. After the appearance of the first disease symptoms, the disease reaction of plants was assessed based on the severity of symptoms as described by Müller and Berg (2008) at weekly intervals for the duration of 7 weeks after inoculation. Data on disease severity was used to calculate area under disease process curve (AUDPC) determined as AUDPC $=\Sigma((\mathrm{Si}+\mathrm{Sti}+1) / 2)^{*}(\mathrm{ti}+1-\mathrm{ti})$, where $\mathrm{Si}$ is the symptoms severity, and ti is the date of assessment of symptoms severity.

\section{Volatile Metabolite Analyses with P. polymyxa Sb3-1 and V. longisporum ELV43}

Antagonism of P. polymyxa Sb3-1 and V. longisporum ELV43 was tested with the "Two Clamp VOCs Assay" as described
A
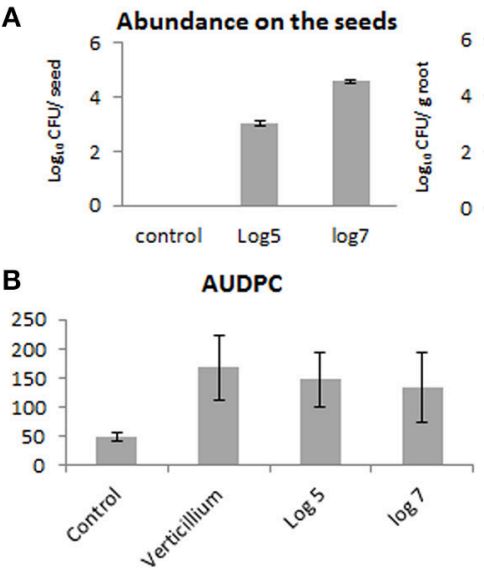
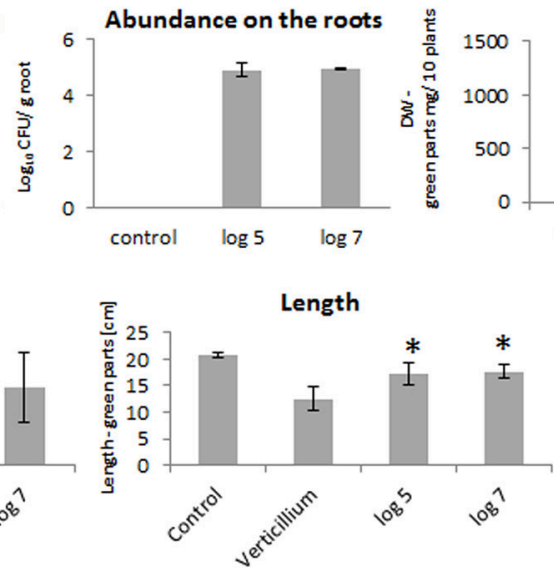

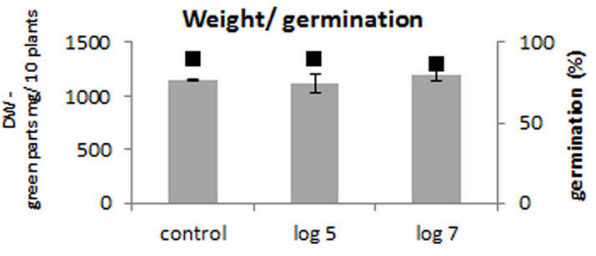

Dry weight

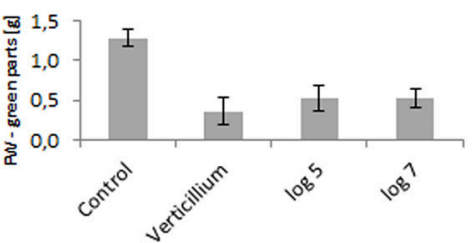

FIGURE 1 | PGP effect on the OSR seedlings and Verticillium wilt disease reduction due to the treatment of the $\mathrm{OSR}$ seeds with $\log _{10} 5$ and log $_{10} 7 \mathrm{CFU}_{\mathrm{ml}}{ }^{-1}$ of P. polymyxa Sb3-1. (A) Abundances of the Sb3-1 on the seeds (left panel) and on the roots (middle panel) as well as fresh weights and germination rates (black squares) of the of the 2-week old seedlings (right panel) treated with Sb3-1 compared to the untreated control (control). (B) The Verticillium wilt disease severity (AUDPC; left panel), and lengths and weights (middle and rights panels, respectively) of the 8 weeks old OSR seedlings infected with $V$. longisporum. The labels of the bars refer to the different concentrations of $P$. polymyxa Sb3-1 in the initial inoculant used for seed treatments: $\log _{5}$, $\log _{10} 5 \mathrm{CFU} \mathrm{ml}^{-1}$ of Sb3-1; log 7 , $\log _{10} 7 \mathrm{CFU}$ $\mathrm{ml}^{-1}$ of Sb3-1; control, untreated control; Verticillium, untreated seedlings that were infected with $\mathrm{V}$. longisporum. Error bars represent confidence interval $(P=0.05)$. The asterisk ( $\left.{ }^{*}\right)$ denotes values that were significantly different from the non-treated control group values $(P<0.05)$ defined using pairwise $t$-test or non-parametric Mann-Whitney $U$-test, depending on the distribution of the samples. 
in Cernava et al. (2015a). The assay was performed in nine replicates. Three plates with three wells each were used per replicate. The significance of the differences between zones of inhibition of Verticillium growth by different bacterial strains (Table 2) was calculated using one-way ANOVA and Tukey's HSD tests. For both analyses, the $P<0.05$ were considered to be significant.

GC-MS headspace SPME experiments were carried out as described by Cernava et al. (2015a; Supplementary Figure 1). Identification of the volatile compounds was performed with the NIST MS Search 2.2 included in the Software-Package of the NIST 2014 database. Further verification was done by calculation of the covats index (CI) followed by comparisons to database entries of NIST Search 2.2 and the entries in the Online Database of NIST (http://webbook.nist.gov/). The raw data of all analyses is provided as Supplementary Data for this publication; file names include the analyzed microorganisms and the duration of the incubation prior to VOC sampling.

\section{Soluble Metabolite Analyses of $P$. polymyxa Sb3-1 and V. longisporum ELV43}

In the course of an LC-MS assay $V$. longisporum ELV43 and $P$. polymyxa Sb3-1 were co-incubated in order to exchange their
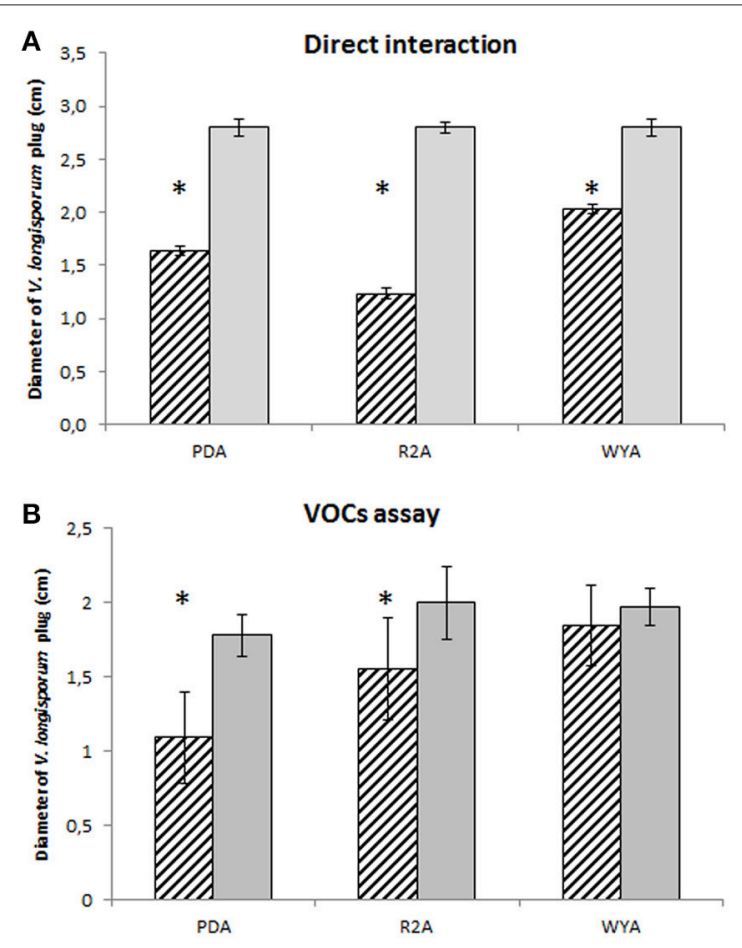

FIGURE 2 | Plate confrontation and VOCs assay of $P$. polymyxa Sb3-1 and $V$. longisporum ELV43 in three different growth media (PDA, R2A, and WYA) for nine days. Dashed columns show growth of $V$. longisporum ELV43 in the presence of $P$. polymyxa Sb3-1 (A) or its VOCs (B), while the gray columns illustrate the growth of $V$. longisporum ELV43 without $P$. polymyxa Sb3-1 (A) or its VOCs (B). The assay was performed in nine replicates. The asterisk ( $\left.{ }^{*}\right)$ denotes values that varied significantly from control group values $(P<0.05)$ defined using pairwise $t$-test or non-parametric Mann-Whitney $U$-test, depending on the distribution of the samples.
VOCs without direct contact with one another. The assay was performed in three replicates. A 4-day old growth plate with $V$. longisporum ELV43 was placed on top of the P. polymyxa Sb31 plate and sealed to facilitate accumulation of VOCs. For the negative control, a fungal and a bacterial plate were incubated with a non-inoculated PDA plate. Cell lysis was performed using Ribolyser FastPrep-24 (MP Biomedicals, Santa Ana, California, USA) for two times $30 \mathrm{~s}$ at $6 \mathrm{~m} \mathrm{~s}^{-1}$ in $90 \%$ methanol. The cell free extract was stored at $-70^{\circ} \mathrm{C}$. The bacterial and fungal metabolite extracts were analyzed with a combined HPLChybrid quadrupole-orbitrap mass spectrometer ( $Q$ Exactive; Thermo Scientific, Bremen, Germany). A Luna 5u NH2 100A $250 \times 4.6$ column (Phenomenex, Aschaffenburg, Germany) was used to separate different metabolites from the cell extracts as described by Cernava et al. (2015b). Identification of the soluble compounds was performed with the XCalibur 2.2 and SIEVE 2.2 (Thermo Scientific, Bremen, Germany) and manual comparison of the spectra with corresponding spectra from literature as well as such from mzCloud (HighChem LLC, Bratislava, Slovakia).

\section{Statistical Analysis}

The PGP and antifungal effects of the Sb3-1 were statistically analyzed using the IBM SPSS program version 20.0 (IBM Corporation, Armonk, NY, USA). The significance of the differences in the assessed features (germination rate, plants' weights, diameter of the Verticillium plugs) between the control vs. each treatment group was calculated using a pairwise $t$-test with independent samples. The decision to make use of the nonparametric Mann-Whitney $U$-test as an alternative to the $t$-test was based an assessment of the distributions of variables (normal vs. non-normal).

\section{RESULTS}

\section{PGP and Antifungal Effects of $P$. polymyxa Sb3-1 Applied to the Seeds of OSR}

The seed treatments with $\log _{10} 5$ and $\log _{10} 7 \mathrm{CFU} \mathrm{ml} \mathrm{m}^{-1}$ of $P$. polymyxa Sb3-1 rif $^{\mathrm{R}}$ resulted in recovery rates of $\log _{10} 3 \pm$ 0.1 and $\log _{10} 4.6 \pm 0.1 \mathrm{CFU}$ seed $^{-1}$, respectively (Figure 1A). The concentration of Sb3-1 in the roots of the 2-week old seedlings was $\log _{10} 4.9 \pm 0.3 \mathrm{CFU} \mathrm{g} \mathrm{root}^{-1}$, independent of the initial inoculum concentration. We observed no PGP effect or effect on the germination rate of the Sb3-1 treatment on the 2 -week old OSR seedlings (Figure 1A). When the 1-week old seedlings were inoculated with $V$. longisporum, we observed a mild improvement in disease resistance and dry weight as well as a significant increase in the respective lengths of the seedlings treated with $P$. polymyxa Sb3-1 rif $^{\mathrm{R}}$ (Figure 1B). The observed increase in seedling length was 36 and $40 \%$ for the treatments with $\log _{10} 5$ and $\log _{10} 7 \mathrm{CFU} \mathrm{m} \mathrm{m}^{-1}$, respectively, compared to the untreated control infested with $V$. longisporum.

\section{P. polymyxa Sb3-1 and Its VOCs Inhibit Growth of V. longisporum ELV43 in the Plate Assay}

In order to understand the mechanism of the antifungal effect of $P$. polymyxa Sb3-1 we designed a series of experiments 
TABLE 1 | GC-MS headspace SPME identification of relevant VOCs produced by P. polymyxa Sb3-1 and V. Iongisporum ELV43 grown for 3 or 6 days in presence of each other volatiles.
RT (min)
Predicted compound ${ }^{a}$
Regulation $^{b}$
Predicted function

\section{VOCs PRODUCED BY Sb3-1 AFTER BOTH 3 AND 6 DAYS GROWTH IN PRESENCE OF V. longisporum VOCs}

\begin{tabular}{|c|c|c|c|}
\hline 8.521 & 1,3-Dioxolane, 2,2,4,5-tetramethyl-, trans-* & $\uparrow 986 \%$ ** & N.a. \\
\hline 12.238 & Pentafluoropropionic acid, hexyl ester* & $\downarrow 47,6 \%$ ** & N.a. \\
\hline 19.248 & 2-Nonanone* & - & Antibacterial (Schulz and Dickschat, 2007) \\
\hline 20.893 & 2-Decanone* & - & N.a. \\
\hline 20.980 & 2-Decanol ${ }^{*}$ & - & N.a. \\
\hline \multicolumn{4}{|c|}{ VOCs PRODUCED BY Sb3-1 ONLY AFTER 3 DAYS OF CO-INCUBATION WITH V. longisporum VOCs } \\
\hline 5.25 & 2,3- Butanedione* & - & $\begin{array}{l}\text { PGP, antibacterial (Farag et al., 2006; Lee et al., } \\
\text { 2012) }\end{array}$ \\
\hline 5.8 & 2-Methyl-1-Propanol* & - & Antifungal (Stotzky et al., 1976) \\
\hline 6.438 & 1-Butanol ${ }^{\star}$ & - & Antibacterial (Létoffé et al., 2014) \\
\hline 7.497 & 3-Hydroxy-2-butanone* & - & PGP, ISR (Ryu et al., 2003) \\
\hline 8.239 & 2-Methyl-1-butanol* & - & Antifungal (Raza et al., 2015) \\
\hline 8.605 & 3-Methyl-2-pentanone* & - & N.a. \\
\hline 12.501 & Isoamyl Acetate & $\downarrow 56 \%$ & Antimicrobial (Ando et al., 2015) \\
\hline 13.335 & 2-Heptanol* & - & Antimicrobial (Orhan et al., 2012) \\
\hline 19.621 & Decane, 2,6,7 trimethyl ${ }^{\star}$ & - & N.a. \\
\hline 20.773 & Hexadecanal & - & Antifungal (Raza et al., 2015) \\
\hline 21.090 & Decyl trifluoroacetate* & - & N.a. \\
\hline 23.091 & Durenol & $\downarrow 34.1 \% \%^{\star \star}$ & Possibly antimicrobial (Al Nomaani et al., 2013) \\
\hline 23.232 & 2-(2-Methylpropyl)-3- (1-methylethyl) pyrazine & - & Antimicrobial (Aichner et al., 2012) \\
\hline 26.556 & Spathulenol & $\downarrow 44 \%$ ** & Plant metabolite (Pacciaroni et al., 2008) \\
\hline 24.686 & 2-Dodecanal* & - & N.a. \\
\hline \multicolumn{4}{|c|}{ VOCs PRODUCED BY Sb3-1 ONLY AFTER 6 DAYS GROWTH IN PRESENCE OF V. Iongisporum VOCs } \\
\hline 10.25 & 2-Hydroxy-3-pentanone* & - & N.a. \\
\hline 12.25 & Pentaflouropropionic acid, hexyl ester* & - & N.a. \\
\hline 15.75 & 2- Isopropylpyrazine & - & Antimicrobial (Aichner et al., 2012) \\
\hline 16.6 & 3(2H)-Thiononanoe & $\uparrow 51.5 \%$ & N.a. \\
\hline 17.79 & 1,2-Butanediol, 1-phenyl & - & N.a. \\
\hline 18.74 & p-cresol* & - & toxic for eukaryotic cells (Andriamihaja et al., 2015) \\
\hline 23.13 & 2- N-(2-methylpropyl) Benzothiazolamine & $\downarrow 38.3 \%$ & N.a. \\
\hline 23.23 & 2-(2-Methylpropyl-)-3-(1-methylethyl) pyrazine & - & Antimicrobial (Aichner et al., 2012) \\
\hline \multicolumn{4}{|c|}{ VOCs PRODUCED BY VL43 AFTER BOTH 3 AND 6 DAYS GROWTH IN PRESENCE OF P. polymyxa Sb3-1 VOCs } \\
\hline 5.80 & |sobutanol* & - & Antifungal (Stotzky et al., 1976) \\
\hline 19.90 & 2-phenylethanol* & - & Antimicrobial (Liu and Zhang, 2015) \\
\hline \multicolumn{4}{|c|}{ VOCs PRODUCED ONLY AFTER 3 DAYS OF CO-INCUBATION WITH P. polymyxa Sb3-1 VOCs } \\
\hline 7.65 & Acetoin* & - & PGP, ISR (Ryu et al., 2003) \\
\hline 13.34 & 2-(4-Cyclohexyl-butanoylamino) -3-chloro-1,4-naphthoquinone & $\uparrow 100 \%$ ** & Putatively antifungal (Sasaki et al., 2002) \\
\hline 17.792 & Bicyclo (2.2.1)-hepta-2,5-dien-7-ol; or 7-Hydroxynorbornadiene & $\uparrow 100 \%$ ** & N.a. \\
\hline \multicolumn{4}{|c|}{ VOCs PRODUCED ONLY AFTER 6 DAYS GROWTH IN PRESENCE OF P. polymyxa Sb3-1 VOCs } \\
\hline 6.44 & 1-Butanol ${ }^{\star}$ & $\uparrow 53.4 \%$ & Antimicrobial (Létoffé et al., 2014) \\
\hline 8.10 & Isoamyl alcohol* & - & Antimicrobial (Ando et al., 2015) \\
\hline 9.23 & 1,2,4- Benzenetricarboxylic acid, 1,2 dimethyl ester & $\uparrow 100 \%$ ** & N.a. \\
\hline 17.793 & $\mathrm{~N} \alpha, \mathrm{N} \omega$-Dicarbobenzoxy-L-arginine & $\uparrow 100 \%{ }^{\star \star}$ & N.a. \\
\hline 18.08 & 2-Nonanone* & - & Antifungal (Raza et al., 2015) \\
\hline 19.98 & 3-Phenyl-5-(benzylthio)isoxazole & $\uparrow 17.9 \%$ & N.a. \\
\hline 25.84 & 5,6-Decadien-3-yne,5,7-diethyl & $\uparrow 20 \%$ & N.a. \\
\hline
\end{tabular}

a The selection of substances included in the Table was performed as followed: (1) only substances with match index with the NIST MS Search 2.2 included in the Software-Package of the NIST 2014 database over 500 were considered; (2) only the substances that were either verified using covats index [<15; labeled with asterisks ( $\left.{ }^{*}\right)$ ] or showed an up- or down-regulation due to the presence of the VOCs of the other microorganism were included in the Table. The predicted functions of the substances are highlighted in color as follows: the substances with predicted antimicrobial activity are shown in light red, substances with putatively plant growth regulating functions are light green and unknown substances are highlighted in gray.

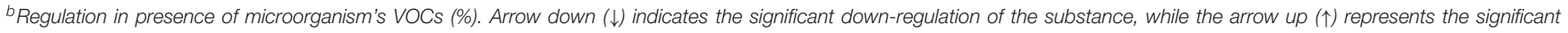

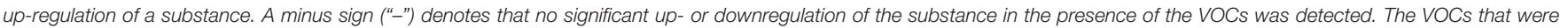
identified by Raza et al. (2015) as produced by P. polymyxa WR-2 are highlighted in bold.

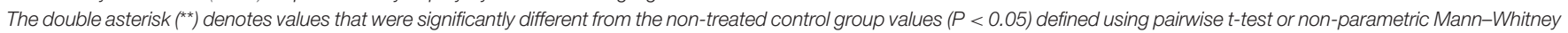
$U$-test, depending on the distribution of the samples. 
TABLE 2 | Effect of $V$. longisporum ELV43 VOCs on P. polymyxa Sb3-1 metabolism detected by LC-MS.

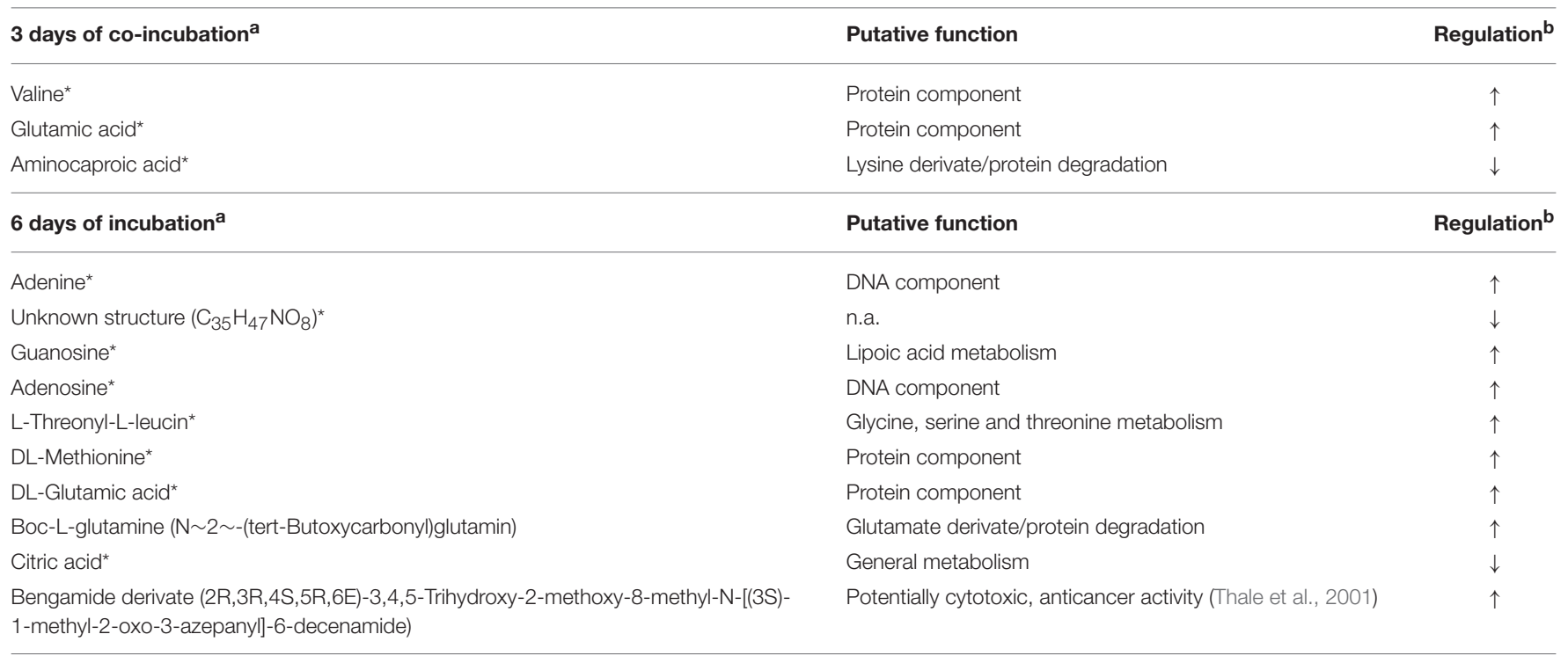

${ }^{a}$ Only verified substances that were up- or down-regulated by V. Iongisporum ELV43 VOCs are shown.

${ }^{b}$ Arrow up $(\uparrow)$ symbolizes that the corresponding substance is upregulated, while arrow down ( $\left.\downarrow\right)$ symbolizes a downregulation by V. Iongisporum ELV43 VOCs. N.a. (not available) means that a putative function could not be found. Asterisk $\left(^{*}\right)$ symbolized that the up- or downregulation was statistically significant $(p<0.05)$.

TABLE 3 | Effect of $P$. polymyxa Sb3-1 VOCs on V. longisporum ELV 43 metabolism detected by LC-MS.

\begin{tabular}{|c|c|c|}
\hline 3 days of co-incubation ${ }^{a}$ & Putative function & Regulation $^{b}$ \\
\hline DL-Histidine* & Protein component & $\downarrow$ \\
\hline DL-Proline ${ }^{*}$ & Protein component & $\downarrow$ \\
\hline DL-Glutamic acid* & $\begin{array}{l}\text { Citric acid cycle/Protein } \\
\text { component }\end{array}$ & $\downarrow$ \\
\hline L-Isoleucyl-L-glutamic acid* & Protein breakdown product & $\uparrow$ \\
\hline Cystathionine ${ }^{*}$ & Cystein synthesis intermediate & $\downarrow$ \\
\hline DL-Glutamine ${ }^{\star}$ & Protein component & $\downarrow$ \\
\hline L-Saccharopine* & Lysine synthesis intermediate & $\downarrow$ \\
\hline DL-2,6-Diaminopimelic acid & Cell wall component & $\downarrow$ \\
\hline Pantothenic acid* & Vitamine & $\downarrow$ \\
\hline 6 days co-incubation ${ }^{a}$ & Putative function & Regulation \\
\hline Arabitol* & Sugar/response to cell stress & $\uparrow$ \\
\hline L-Isoleucyl-L-glutamic acid & Protein breakdown product & $\uparrow$ \\
\hline Methylglutaric acid ${ }^{\star}$ & n.a. & $\uparrow$ \\
\hline Uridine* $^{*}$ & RNA component & $\uparrow$ \\
\hline N-Acetyl-L-Carnosine & Antioxidant & $\downarrow$ \\
\hline Cystathionine $^{\star}$ & $\begin{array}{l}\text { Intermediate in the synthesis of } \\
\text { cysteine }\end{array}$ & $\downarrow$ \\
\hline
\end{tabular}

anly verified substances that were up- or down-regulated by V. Iongisporum ELV43 VOCs are shown.

${ }^{b}$ Arrow up $(\uparrow)$ symbolizes that the corresponding substance is upregulated, while arrow down ( $\downarrow$ ) symbolizes a down regulation. n.a. (not available) means that a putative function could not be found. Asterisk (*) symbolized that the up- or downregulation was statistically significant $(p<0.05)$.

demonstrating the interaction between $P$. polymyxa Sb3-1 and $V$. longisporum ELV43 in vitro on a dual-plate assay and using a specific VOCs assay. After 9 days of co-inoculation the mycelial growth of $V$. longisporum ELV43 was significantly inhibited by $P$. polymyxa Sb3-1 on all media tested (Figure 2A). The incubation of $V$. longisporum ELV43 with the VOCs of Sb3-1 resulted in the significant inhibition of mycelium growth only in combination with PDA and R2A as growth media (Figure 2B). The strongest inhibition of the mycelia growth by $P$. polymyxa VOCs was observed when both microorganisms were grown on PDA plates.

\section{VOCs Involved in the Interaction between P. polymyxa Sb3-1 and V. longisporum ELV43}

VOCs produced by $P$. polymyxa Sb3-1 after 3 and 6 days of coincubation with $V$. longisporum ELV43 volatiles were analyzed using GC-MS headspace SPME, compared to database entries (NIST 2014 database), and verified using covats index (CI; Table 1). Several substances with strong antimicrobial effect, such as three alkyl-substituted pyrazines (Cernava, 2012) were found to be produced after either 3 or 6 days of bacterial growth regardless of the interaction with ELV43 VOCs. We found that higher quantities of known antimicrobial VOCs, such as 2methyl-1-butanol, hexadecanal (Raza et al., 2015), and isoamyl acetate (Ando et al., 2015), were produced by Sb3-1 after 3 days of incubation than after 6 days of incubation (Table 1). The putatively antimicrobial substances isoamyl acetate and durenol were both downregulated upon contact with $V$. longisporum ELV43 VOCs. We also found two CI-verified substances, 2,3-butanedione and 3-hydroxy-2-butanone 1 within VOCs produced by Sb3-1 that are putatively involved in a bacterial PGP effect (Ryu et al., 2003; Farag et al., 2006). Neither of them was regulated by $V$. longisporum ELV43 VOCs (Table 1).

GC-MS headspace SPME analysis revealed that $V$. longisporum ELV43 produced several substances with 
known antimicrobial properties such as isobutanol (Stotzky et al., 1976) and 2-phenylethanol (Liu and Zhang, 2015). These substances were detected after 3 and 6 days of V. longisporum growth. Acetoin, a substance with a known PGP effect that also induces systemic resistance and regulates auxin homeostasis in A. thaliana (Ryu et al., 2003), was detected in the volatile phase after 3 days of $V$. longisporum growth. Seven VOCs were upregulated in ELV43 during co-incubation with $P$. polymyxa Sb3-1 volatiles (Table 1). Two of them, a naphthoquinonederivate and 1-Butanol, both exhibiting putative antimicrobial effect (Sasaki et al., 2002; Létoffé et al., 2014) were upregulated due to contact with Sb3-1 VOCs (100 and 53.4\%, respectively).

\section{Regulation of Soluble Metabolites Produced by $P$. polymyxa Sb3-1 and V. longisporum Elv43 as a Reaction to Each Other VOCs}

The production of soluble metabolites by both microorganisms, $P$. polymyxa Sb3-1 and V. longisporum ELV43 in response to one another's VOCs was studied by means of high-resolution LC-MS analyses. More than 100 substances were detected in the P. polymyxa Sb3-1 samples (data not shown). Of the substances that we were able to be identified with a high degree of certainty (based on NIST 2014 database searches), several were differentially regulated due to the interaction with ELV43 VOCs. Two amino acids, valine and glutamic acid, were upregulated in the 3-day old culture of Sb3-1 due to its exposure to $V$. longisporum EVL43 volatiles, while one amino acid derivative, aminocaproic acid, was downregulated (Table 2). Six days after inoculation, several substances involved in the cellular metabolism, such as amino- and nucleic acids were upregulated. Only two substances, citric acid and an

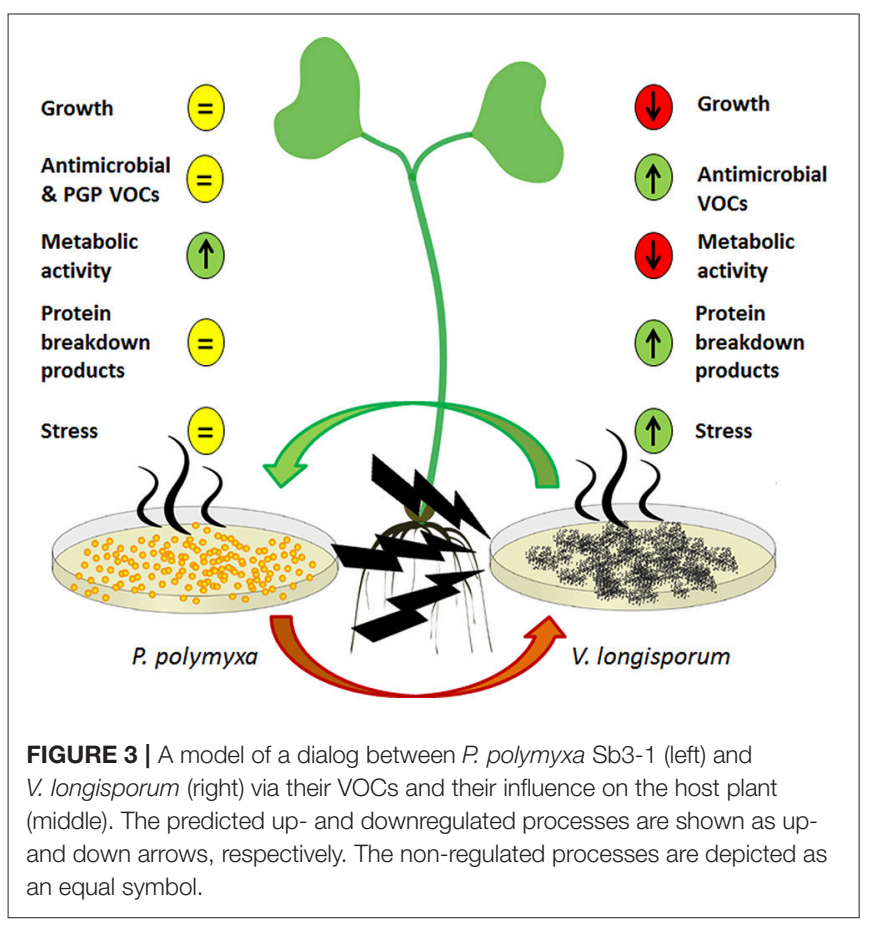

unidentified substance, were downregulated because of Sb3-1 exposure to ELV43 VOCs. Additionally, surfactin was detected in every sample of $P$. polymyxa Sb3-1 regardless of the EVL43 VOCs exposure. Surfactin is known for its biosurfactant and antibacterial properties (Pratap et al., 2013) and for its ability to activate the formation of biofilm (Timmusk et al., 2015). Spermidine, a stress protection agent (Kusano et al., 2008), was found after 6 days of Sb3-1 growth, while antimicrobial fusaricidin (Kajimura and Kaneda, 1996) was found after both 3 and 6 days of bacterial growth. Both components were not regulated by EVL43 VOCs. Interestingly, other wellknown antimicrobial Paenibacillus metabolite polymyxin was not detected under the conditions tested in any of the samples.

Analysis of $V$. longisporum ELV43 metabolites showed that 3 days of incubation with $P$. polymyxa Sb3-1 VOCs lead to up-regulation of L-Isoleucyl-L-glutamic acid, an incomplete breakdown product of protein digestion. On the other hand, the concentration of several metabolites involved in cellular metabolism (e.g., several amino acids and intermediates in the amino acid synthesis), of a substance involved in the cell wall formation (DL-2,6-Diaminopimelic acid) as well as of pantothenic acid, decreased (Table 3 ). Interestingly, after 6 days of co-incubation with P. polymyxa Sb3-1 volatiles, the upregulation of detected substances prevailed over downregulation. We detected upregulation of arabitol, a carbohydrate involved in cell stress response, and several breakdown products of protein and RNA biosynthesis. On the other hand, N-Acetyl-L-Carnosine (known as an antioxidant) and cystathionine were downregulated following exposure to Sb3-1 VOCs.

\section{DISCUSSION}

The current study underpins the importance of volatiles as mediators of microbial interactions. Moreover, the results suggest that microbial VOCs from beneficial bacteria can induce substantial changes in target pathogens. P. polymyxa Sb3-1 was able to reduce the growth of $V$. longisporum ELV43 in vitro directly, and via its volatiles. The antifungal effect of Sb31 was confirmed in planta by a significant improvement in the lengths of the oilseed rape seedlings treated with Sb3-1 compared to the untreated control. The observed plant growth promotion effect was only detected when the seedlings were infested with $V$. longisporum. This suggests that the antagonistic properties of the Paenibacillus strain or its ability to induce resistance in the host plant were responsible for the beneficial effect on the plant growth. The production of a biosurfactant and activator of biofilm formation surfactin (Pratap et al., 2013; Timmusk et al., 2015) as well as the stress protection agent spermidine (Kusano et al., 2008) and antimicrobial fusaricidin by Sb3-1 may enhance the PGP effect under biotic stress conditions. The production of 2,3-butanedione and 3-hydroxy2-butanone by Sb3-1 may also explain PGP properties of Sb31 observed in our greenhouse experiments and in previous in planta tests under sterile soil conditions (Rybakova et al., 2016b). 
The majority of our results revealed contrasting effects with regards to the ways in which the two microorganisms reacted to one another's VOCs as illustrated by the model in Figure 3. While $P$. polymyxa Sb3-1 was not visually impaired in its growth due to ELV43 volatiles, the growth rate of $V$. longisporum ELV43 was significantly reduced. Both of the microorganisms constantly produced potentially antimicrobial volatiles; however the production of some antimicrobial VOCs by Sb3-1 was reduced upon contact with ELV43 VOCs, while ELV43 demonstrated upregulation of antimicrobial VOCs due to the interaction with Sb3-1. This may indicate that P. polymyxa does not "regard" $V$. longisporum as an enemy and slows its defense system down, while the fungus switches on some type of additional defense mechanism to counterattack the antimicrobial VOCs of Sb3-1. A similar effect was observed when we studied soluble metabolites of both interacting microorganisms. While $P$. polymyxa Sb3-1 demonstrated upregulation of some essential amino acids and DNA components, the opposite was found for $V$. longisporum ELV43. This finding may reflect a change in the metabolic rate of both microorganisms as a reaction to one another's VOCs. There are indications that the metabolism of $V$. longisporum ELV43 slows down due to the contact with damaging Sb3-1 VOCs as the cells undergo an apoptotic process. This was also confirmed by the observed accumulation of protein breakdown products and a specific carbohydrate indicating cellular stress (arabitol) by ELV43 exposed to Sb3-1 VOCs. In contrast, the metabolic activity of Sb3-1 seems to be activated by the ELV43 VOCs as is suggested by accumulation of protein and DNA components by ELV43.

Interestingly, out of 40 identified VOCs produced by $P$. polymyxa Sb3-1 only three (2-nonanone, 2-methyl-1-butanol, and hexadecanal) were identical with those identified by Raza et al. (2015) in P. polymyxa WR-2 VOCs. This strain was found to be active against Fusarium oxysporum f. sp. niveum in vitro. The variations in the composition of volatiles might be primarily due to differences in the growth media compositions, however the structure of Paenibacillus' "volatilomes" can also vary to a certain degree as highlighted by Rybakova et al. (2016a). In addition, it indicates a very specific pathogen-antagonist interaction. We first detected one putative PGP substance acetoin (3-hydroxy2-butanone), which was also found to trigger induced systemic resistance in Arabidopsis (Rudrappa et al., 2010) produced by the plant pathogen $V$. longisporum ELV43. Although, this finding needs to be further investigated, it may also explain the plantbeneficial effect described for non-pathogenic Verticillium strains (França et al., 2013). Furthermore, it also supports the theory that microbial diversity is crucial for combating "microbiome diseases" such as Verticillium wilt because Verticillium species

\section{REFERENCES}

Aichner, M., Oberauner, L., Liebminger, S., Fürnkranz, M., Klein, T., Khinast, J., et al. (2012). Volatile Organic Compounds from Bacterial Antagonists for Controlling Microbial Growth. Patent WO 2012085255 A2. occur frequently in healthy plants and contribute to the functioning of the holo-biont (Berg et al., 2017).

In conclusion, our data indicates that $P$. polymyxa Sb3-1 and $V$. longisporum ELV43 are in a constant dialog with one another via their VOCs. This specific dialog results in the inhibition of cellular metabolism in $V$. longisporum ELV43 leading to the growth reduction of the fungus. This antagonistic effect in addition to the production of PGP volatiles leads to an overall positive effect on the growth of the infested host plant. This study contributes to the better overall understanding of the interactions between the potential BCA P. polymyxa Sb3-1 and the plant pathogen $V$. longisporum. It supports the effort to develop this beneficial bacterium into a viable BCA against Verticillium wilt, and offers a promising option in the progress toward realizing sustainable agriculture in the context of global warming.

\section{AUTHOR CONTRIBUTIONS}

GB and DR designed the study. UR carried out the in vitro, LCMS and GC-MS assays and analyzed the GC-MS data. MS and DR carried out plant growth promoting and biocontrol assays. AS did the analytic for LC-MS assays. TC and AS consulted on the GCMS assays and TC contributed to the VOCs-related parts of the final manuscript. DR wrote the final version of the manuscript with input from GB, TC, and UR. All authors read and approved the final manuscript.

\section{ACKNOWLEDGMENTS}

This project was funded by the European Union in frame of FP7-KBBE-2013-7-single-stage (BIOCOMES; No. 612713) and by the Austrian Research Promotion Agency (FFG; No. 836466). TC's involvement has been supported by the Federal Ministry of Science, Research and Economy (BMWFW), the Federal Ministry of Traffic, Innovation and Technology (bmvit), the Styrian Business Promotion Agency SFG, the Standortagentur Tirol, the Government of Lower Austria and ZIT-Technology Agency of the City of Vienna through the COMET-Funding Program managed by the $12 \mathrm{FFG}$ (grant number 282482). The authors gratefully acknowledge support from NAWI Graz. The authors would like to thank Timothy Mark (Graz) for English revision and discussion.

\section{SUPPLEMENTARY MATERIAL}

The Supplementary Material for this article can be found online at: http://journal.frontiersin.org/article/10.3389/fpls.2017. 01294/full\#supplementary-material 
Ando, H., Kurata, A., and Kishimoto, N. (2015). Antimicrobial properties and mechanism of volatile isoamyl acetate, a main flavour component of Japanese sake (Ginjo-shu). J. Appl. Microbiol. 118, 873-880. doi: 10.1111/jam.12764

Andriamihaja, M., Lan, A., Beaumont, M., Audebert, M., Wong, X., Yamada, K., et al. (2015). The deleterious metabolic and genotoxic effects of the bacterial metabolite p-cresol on colonic epithelial cells. Free Radic. Biol. Med. 85, 219-227. doi: 10.1016/j.freeradbiomed.2015.04.004

Baldwin, I. T., Halitschke, R., Paschold, A., Von Dahl, C. C., and Preston, C. A. (2006). Volatile signaling in plant-plant interactions: "talking trees" in the genomics era. Science 311, 812-815. doi: 10.1126/science.1118446

Berg, G., Köberl, M., Rybakova, D., Müller, H., Grosch, R., and Smalla, K. (2017). Plant microbial diversity is suggested as the key to future biocontrol and health trends. FEMS Microbiol. Ecol. 93:fix050. doi: 10.1093/femsec/fix050

Cernava, T. (2012). Identification of Volatile Organic Compounds from PlantAssociated Bacteria. Master thesis, Graz University of Technology, Graz, AU.

Cernava, T., Aschenbrenner, I. A., Grube, M., Liebminger, S., and Berg, G. (2015a). A novel assay for the detection of bioactive volatiles evaluated by screening of lichen-associated bacteria. Front. Microbiol. 6:398. doi: $10.3389 /$ fmicb. 2015.00398

Cernava, T., Müller, H., Aschenbrenner, I. A., Grube, M., and Berg, G. (2015b). Analyzing the antagonistic potential of the lichen microbiome against pathogens by bridging metagenomic with culture studies. Front. Microbiol. 6:620. doi: 10.3389/fmicb. 2015.00620

Daebeler, F., Amelung, D., and Zeise,. K. (1988). Verticillium-welke an winterraps-auftreten und bedeutung. Nachrichtenblatt Pflanzenschutzdienst DDR 42, 71-73.

Depotter, J. R., Deketelaere, S., Inderbitzin, P., Tiedemann, A. V., Höfte, M., Subbarao, K. V., et al. (2016). Verticillium longisporum, the invisible threat to oilseed rape and other brassicaceous plant hosts. Mol. Plant Pathol. 17, 1004-1016. doi: 10.1111/mpp. 12350

Dicke, M., van Loon, J. J. A., and Soler, R. (2009). Chemical complexity of volatiles from plants induced by multiple attack. Nat. Chem. Biol. 5, 317-324. doi: $10.1038 /$ nchembio. 169

Ditengou, F. M., Müller, A., Rosenkranz, M., Felten, J., Lasok, H., van Doorn Miloradovic, M., et al. (2015). Volatile signaling by sesquiterpenes from ectomycorrhizal fungi reprogrammes root architecture. Nat. Commun. 6:6279. doi: $10.1038 /$ ncomms 7279

Dunker, S., Keunecke, H., Steinbach, P., and Von Tiedemann, A. (2008). Impact of Verticillium longisporum on yield and morphology of winter oilseed rape (Brassica napus) in relation to systemic spread in the plant. J. Phytopathol. 156, 698-707. doi: 10.1111/j.1439-0434.2008. 01429.x

Effmert, U., Kalderas, J., Warnke, R., and Piechulla, B. (2012). Volatile mediated interactions between bacteria and fungi in the soil. J. Chem. Ecol. 38, 665-703. doi: 10.1007/s10886-012-0135-5

Farag, M. A., Ryu, C. M., Sumner, L. W., and Paré, P. W. (2006). GCMS SPME profiling of rhizobacterial volatiles reveals prospective inducers of growth promotion and induced systemic resistance in plants. Phytochemistry 67, 2262-2268. doi: 10.1016/j.phytochem.2006. 07.021

Fradin, E. F., Zhang, Z., Ayala, J. C., Castroverde, C. D., Nazar, R. N., Robb, J., et al. (2009). Genetic dissection of Verticillium wilt resistance mediated by tomato Ve1. Plant Physiol. 150, 320-332. doi: 10.1104/pp.109.136762

França, S. C., Spiessens, K., Pollet, S., Debode, J., De Rooster, L., Callens, D., et al. (2013). Population dynamics of Verticillium species in cauliflower fields: influence of crop rotation, debris removal and ryegrass incorporation. Crop Protect. 54, 134-141. doi: 10.1016/j.cropro.2013.08.008

Grady, E. N., MacDonald, J., Liu, L., Richman, A., and Yuan, Z. C. (2016). Current knowledge and perspectives of Paenibacillus: a review. Microb. Cell Fact. 15:203. doi: 10.1186/s12934-016-0603-7

Hacquard, S. (2017). Commentary: microbial small talk: volatiles in fungalbacterial interactions. Front. Microbiol. 8:1. doi: 10.3389/fmicb.2017.00001

Heale, J. B., and Karapapa, V. K. (1999). The verticillium threat to canada's major oilseed crop: canola. Can. J. Plant Pathol. 21, 1-7. doi: 10.1080/07060661.1999.10600114

Hiemstra, J. A. (1998). A Compendium of Verticillium Wilts in Tree Species. Wageningen: CPRO.
Hol, W. H., Garbeva, P., Hordijk, C., Hundscheid, M. P., Gunnewiek, P. J., Van Agtmaal, M., et al. (2015). Non-random species loss in bacterial communities reduces antifungal volatile production. Ecology 96, 2042-2048. doi: 10.1890/14-2359.1

Houghton, J. T., Ding, Y., Griggs, D. J., Noguer, M., van der Linden, P. J., Dai, X., et al. (2001). Climate Change 2001: The Scientific Basis. The Press Syndicate of the University of Cambridge.

Howden, S. M., Soussana, J. F., Tubiello, F. N., Chhetri, N., Dunlop, M., and Meinke, H. (2007). Adapting agriculture to climate change. Proc. Natl. Acad. Sci. U.S.A. 104, 19691-19696. doi: 10.1073/pnas.0701890104

Insam, H., and Seewald, M. S. A. (2010). Volatile organic compounds (VOCs) in soils. Biol. Fertil. Soils 46, 199-213. doi: 10.1007/s00374-010-0442-3

Jiménez-Gasco, M. D. M., Malcolm, G. M., Berbegal, M., Armengol, J., and Jiménez-Díaz, R. M. (2014). Complex molecular relationship between vegetative compatibility groups (VCGs) in Verticillium dahliae: VCGs do not always align with clonal lineages. Phytopathology 104, 650-659. doi: 10.1094/PHYTO-07-13-0180-R

Kai, M., Effmert, U., Berg, G., and Piechulla, B. (2007). Volatiles of plant growth promoting rhizobacteria (PGPR) influence mycelial growth of the fungus Rhizoctonia solani. Arch. Microbiol. 187, 351-360. doi: 10.1007/s00203-006-0199-0

Kajimura, Y., and Kaneda, M. (1996). Fusaricidin A, a new depsipeptide antibiotic produced by Bacillus polymyxa KT-8. J. Antibiot. 49, 129-135. doi: 10.7164/antibiotics.49.129

Kanchiswamy, C. N., Malnoy, M., and Maffei, M. E. (2015). Chemical diversity of microbial volatiles and their potential for plant growth and productivity. Front. Plant Sci. 6:151. doi: 10.3389/fpls.2015.00151

Karapapa, V. K., Bainbridge, B. W., and Heale, J. B. (1997). Morphological and molecular characterization of Verticillium longisporum comb. nov. pathogenic to oilseed rape. Mycol. Res. 101, 1281-1294. doi: 10.1017/S095375629 7003985

Köberl, M., Ramadan, E. M., Adam, M., Cardinale, M., Hallmann, J., Heuer, H., et al. (2013). Bacillus and Streptomyces were selected as broad-spectrum antagonists against soilborne pathogens from arid areas in Egypt. FEMS Microbiol. Lett. 342, 168-178. doi: 10.1111/1574-6968.12089

Kusano, T., Berberich, T., Tateda, C., and Takahashi, Y. (2008). Polyamines: essential factors for growth and survival. Planta 228, 367-381. doi: 10.1007/s00425-008-0772-7

Lee, B., Farag, M. A., Park, H. B., Kloepper, J. W., Lee, S. H., and Ryu, C. M. (2012). Induced resistance by a long-chain bacterial volatile: elicitation of plant systemic defense by a C13 volatile produced by Paenibacillus polymyxa. PLoS ONE 7:e48744. doi: 10.1371/journal.pone.0048744

Létoffé, S., Audrain, B., Bernier, S. P., Delepierre, M., and Ghigo, J. M. (2014). Aerial exposure to the bacterial volatile compound trimethylamine modifies antibiotic resistance of physically separated bacteria by raising culture medium pH. MBio 5, e00944-e00913. doi: 10.1128/mBio.00944-13

Liu, X. M., and Zhang, H. (2015). The effects of bacterial volatile emissions on plant abiotic stress tolerance. Front. Plant Sci. 6:774. doi: 10.3389/fpls.2015.00774

Messner, R., Schweigrofler, W., Ibl, M., Berg, G., and Prillinger, H. (1996). Molecular characterization of the plant pathogen Verticillium dahliae Kleb. using RAPD-PCR and sequencing of the 18 S rRNA-gene. J. Phytopathol. 144, 347-354. doi: 10.1111/j.1439-0434.1996.tb00305.x

Müller, H., and Berg, G. (2008). Impact of formulation procedures on the effect of the biocontrol agent Serratia plymuthica HRO-C48 on Verticillium wilt in oilseed rape. Biocontrol 53, 905-916. doi: 10.1007/s10526-007-9111-3

Orhan, I. E., Özçelik, B., Kartal, M., and Kan, Y. (2012). Antimicrobial and antiviral effects of essential oils from selected Umbelliferae and Labiatae plants and individual essential oil components. Turk. J. Biol. 36, 239-246. doi: 10.3906/biy-0912-30

Ossowicki, A., Jafra, S., and Garbeva, P. (2017). The antimicrobial volatile power of the rhizospheric isolate Pseudomonas donghuensis P482. PLoS ONE 12:e0174362. doi: 10.1371/journal.pone.0174362

Pacciaroni, A. D., de los Angeles Gette, M., Derita, M., Ariza-Espinar, L., Gil, R. R., Zacchino, S. A., et al. (2008). Antifungal activity of Heterothalamus alienus metabolites. Phytother. Res. 22, 524-528. doi: 10.1002/ptr.2380

Pegg, G. F., and Brady, B. L. (2002). Verticillium Wilts. Wallingford: CABI. doi: $10.1079 / 9780851995298.0000$ 
Pratap, S. S., Seema, G., Neetu, P., and Nimisha, S. (2013). Surfactin: a review on novel microbial surfactant. Int. J. Bioassays 2, 740-745. doi: 10.21746/ijbio.2013.05.001

Raza, W., Yuan, J., Ling, N., Huang, Q., and Shen, Q. (2015). Production of volatile organic compounds by an antagonistic strain Paenibacillus polymyxa WR-2 in the presence of root exudates and organic fertilizer and their antifungal activity against Fusarium oxysporum f. sp. niveum. Biol. Control 80, 89-95. doi: 10.1016/j.biocontrol.2014.09.004

Reinke, J., and Berthold, G. (1879). Die Zersetzung der Kartoffel durch Pilze, Vol. 1. eds. Wiegandt, Hempel, and Parey (Berlin: P. Parey).

Root, T. L., Price, J. T., Hall, K. R., Schneider, S. H., Rosenzweig, C., and Pounds, J. A. (2003). Fingerprints of global warming on wild animals and plants. Nature 421, 57-60. doi: 10.1038/nature01333

Rudrappa, T., Biedrzycki, M. L., Kunjeti, S. G., Donofrio, N., Czymmek, K. J., Paré, P. W., et al. (2010). The rhizobacterial elicitor acetoin induces systemic resistance in Arabidopsis thaliana. Commun. Integr. Biol. 3, 130-138. doi: $10.4161 /$ cib.3.2.10584

Rybakova, D., Cernava, T., Köberl, M., Liebminger, S., Etemadi, M., and Berg, G. (2016a). Endophytes-assisted biocontrol: novel insights in ecology and the mode of action of Paenibacillus. Plant Soil 405, 125-140. doi: 10.1007/s11104-015-2526-1

Rybakova, D., Schmuck, M., Wetzlinger, U., Varo-Suarez, A., Murgu, O., Müller, H., et al. (2016b). Kill or cure? The interaction between endophytic Paenibacillus and Serratia strains and the host plant is shaped by plant growth conditions. Plant Soil 405, 65-79. doi: 10.1007/s11104-015$2572-8$

Ryu, C. M., Farag, M. A., Hu, C. H., Reddy, M. S., Wei, H. X., Paré, P. W., et al. (2003). Bacterial volatiles promote growth in Arabidopsis. Proc. Natl. Acad. Sci. U.S.A. 100, 4927-4932. doi: 10.1073/pnas.0730 845100

Sasaki, K., Abe, H., and Yoshizaki, F. (2002). in vitro antifungal activity of naphthoquinone derivatives. Biol. Pharm. Bull. 25, 669-670. doi: $10.1248 / \mathrm{bpb} .25 .669$

Schmidt, R., Cordovez, V., deBoer, W., Raaijmakers, J., and Garbeva, P. (2015). Volatile affairs in microbial interactions. ISME J. 9, 2329-2335. doi: 10.1038 /ismej.2015.42

Schubert, P., Golldack, J., Schwärzel, H., and Lentzsch, P. (2009). Influence of soil temperature to the pathosystem strawberry-verticillium. Acta Hortic. 838, 139-144. doi: 10.17660/ActaHortic.2009.838.23

Schulz, S., and Dickschat, J. S. (2007). Bacterial volatiles: the smell of small organisms. Nat. Prod. Rep. 24, 814-842. doi: 10.1039/b507392h

Schulz-Bohm, K., Zweers, H., de Boer, W., and Garbeva, P. (2015). A fragrant neighborhood: volatile mediated bacterial interactions in soil. Front. Microbiol. 6:1212. doi: $10.3389 /$ fmicb.2015.01212
Siebold, M., and von Tiedemann, A. (2012). Potential effects of global warming on oilseed rape pathogens in Northern Germany. Fungal Ecol. 5, 62-72. doi: 10.1016/j.funeco.2011.04.003

Stotzky, G., Schenck, S., and Papavizas, G. C. (1976). Volatile organic compounds and microorganisms. CRC Crit. Rev. Microbiol. 4, 333-382. doi: $10.3109 / 10408417609102303$

Thale, Z., Kinder, F. R., Bair, K. W., Bontempo, J., Czuchta, A. M., Versace, R. W., et al. (2001). Bengamides revisited: new structures and antitumor studies. J. Org. Chem. 66, 1733-1741. doi: 10.1021/jo001380+

Timmusk, S., Grantcharova, N., and Wagner, E. G. H. (2005). Paenibacillus polymyxa invades plant roots and forms biofilms. Appl. Environ. Microbiol. 71 7292-7300. doi: 10.1128/AEM.71.11.7292-7300.2005

Timmusk, S., Kim, S.-B., Nevo, E., El Daim, I. A., Ek, B., Bergquist, J., et al. (2015). Sfp-type PPTase inactivation promotes bacterial biofilm formation and ability to enhance wheat drought tolerance. Front. Microbiol. 6:387. doi: $10.3389 /$ fmicb.2015.00387

Wenke, K., Kai, M., and Piechulla, B. (2010). Belowground volatiles facilitate interactions between plant roots and soil organisms. Planta 231, 499-506. doi: 10.1007/s00425-009-1076-2

Zeise, K., and Steinbach, P. (2004). Schwarze rapswurzeln und der vormarsch der verticillium-rapswelke. Raps 22, 170-174. Available online at: http://www.dlgagrofoodmedien.de/raps.html

Zeise, K., and von Tiedemann, A. (2002). Host specialization among vegetative compatibility groups of Verticillium dahliae in relation to Verticillium longisporum. J. Phytopathol. 150, 112-119. doi: 10.1046/j.1439-0434.2002.00730.x

Zhang, Y., Chen, W., Smith, S. L., Riseborough, D. W., and Cihlar, J. (2005). Soil temperature in Canada during the twentieth century: complex responses to atmospheric climate change. J. Geophys. Res. 110:D03112. doi: 10.1029/2004JD004910

Zhang, Z., and Pawliszyn, J. (1993). Headspace solid-phase microextraction. Anal. Chem. 65, 1843-1852. doi: 10.1021/ac00062a008

Conflict of Interest Statement: The authors declare that the research was conducted in the absence of any commercial or financial relationships that could be construed as a potential conflict of interest.

Copyright $\odot 2017$ Rybakova, Rack-Wetzlinger, Cernava, Schaefer, Schmuck and Berg. This is an open-access article distributed under the terms of the Creative Commons Attribution License (CC BY). The use, distribution or reproduction in other forums is permitted, provided the original author(s) or licensor are credited and that the original publication in this journal is cited, in accordance with accepted academic practice. No use, distribution or reproduction is permitted which does not comply with these terms. 